\title{
Comparison of the Efficacy of Two Microsphere Embolic Agents for Transcatheter Arterial Chemoembolization in Hepatocellular Carcinoma Patients
}

\author{
Shao-Hua Lee, MD \\ Chia-Ying Lin, MD \\ Ya-Chun Hsu, MD \\ Yi-Sheng Liu, MD \\ Ming-Tsung Chuang, MD \\ Ming-Ching Ou, MD
}

Department of Medical Imaging,

National Cheng Kung University Hospital, College of Medicine, National Cheng Kung

University, Tainan, Taiwan

\begin{abstract}
Purpose
Transarterial chemoembolization (TACE) delivers cytotoxic drugs intra-arterially and induces ischemic necrosis by arterial embolization. Embolization is achieved using a variety of agents that differ widely in particle size and range, deformation, and in vivo arterial distribution. The clinical significance of these differences has not been thoroughly characterized. The present study is to compare the efficacy of Embosphere and Embozene microspheres in TACE therapy for hepatocellular carcinoma.
\end{abstract}

\section{Materials and Methods}

This retrospective study includes 108 hepatocellular carcinoma (HCC) patients who received TACE/doxorubicin with Embozene (70 patients) or Embosphere (38 patients) at a single medical center. Patient outcomes, including liver function, tumor size, tumor response, and complications after treatment, were analyzed. The change in total target lesion size and tumor response was evaluated according to embolization agent and clinical characteristics.

\section{Results}

The postoperative glutamate oxaloacetate transaminase (mean, 194.5 vs. 147.5; $p=0.032$ ) and bilirubin (mean, $1.11 \mathrm{mg} / \mathrm{dL}$ vs. $0.73 \mathrm{mg} / \mathrm{dL} ; \mathrm{p}=0.016$ ) were higher among patients treated with Embozene, the decrease in the number $(55.86 \pm 25.55 \%$ vs. $41.81 \pm 38.51 \%$, $p=0.027)$ and size $(56.37 \pm 25.91 \mathrm{~mm}$ vs. $43.44 \pm 37.89 \mathrm{~mm}, \mathrm{p}=0.001)$ of liver tumors relative to baseline was greater in these patients than in those treated with Embosphere. These greater antitumor effects were achieved using lower doses of doxorubicin than for treatment with Embozene. Minor complications were more common among patients treated with Embosphere than with Embozene.

\section{Conclusion}

These results suggest that Embozene is more efficacious than Embosphere for HCC treatment using TACE/doxorubicin.

E-mail: emilialiar@yahoo.com.tw

\section{Introduction}

Hepatocellular carcinoma (HCC), the most common hepatic malignancy, has a high mortality rate. Improvements in surgical techniques and growing acceptance of organ transplantation have made liver transplantation a feasible, curative treatment for HCC. However, the shortage of available organs limits transplantation to $30 \%$ of patients who meet the criteria [1]. For this reason, most HCC patients are treated with palliative therapies.

Transarterial chemoembolization (TACE), an intra-arterial catheter-based chemotherapy, selectively delivers high doses of cytotoxic drugs to the tumor bed together with the induction of ischemic necrosis via arterial embolization [2]. This treatment is one of the preferred modalities for HCC patients who are not suitable for curative therapy [3] and is the standard of care for non-surgical patients with tumors limited to 
the liver because it preserves liver function $[4,5]$. TACE also is recommended for late-stage HCC as the first option for palliative treatment. The most common sole-agent anticancer drug used in published TACE studies is doxorubicin (36\%), followed by cisplatin (31\%), epirubicin $(12 \%)$, mitoxantrone $(8 \%)$, mitomycin $(8 \%)$, and SMANCS (5\%) [6].

Transcatheter vascular occlusion is achieved using a variety of embolic agents, including biodegradable products such as gelatin sponges, starch microspheres, and collagen particles for temporary occlusion and non-biodegradable products such as polyvinyl alcohol (PVA) polymer agents, including Embosphere and Embozene, for permanent occlusion [2]. PVA agents vary widely with respect to compressibility, elasticity, and rigidity, with these properties playing critical roles in the level of embolization achieved [7].

Embosphere is a polymeric microsphere made of trisacryl cross-linked to gelatin. This product comes in a variety of calibrated size ranges. Embosphere does not aggregate, has a smooth and hydrophilic surface, and is deformable, allowing it to penetrate deeper and embolize smaller vessels than other products of this type [8]. Embozene microspheres are composed of a hydrogel core (polymethylmethacrylate) and a Polyzene-F shell. These microspheres have a narrower granulometric distribution than the other available microspheres $(100,250,400,500,700$, and $900 \mu \mathrm{m})$, with each size tightly calibrated to have $95 \%$ of the particles within $50 \mu \mathrm{m}$ of the nominal size. Embozene microspheres have a higher in vivo deformation, which results in more distal occlusion within the vascular network as compared to that of Embosphere microspheres of medium to large size [9]. However, it remains to be determined whether these theoretical advantages of Embozene bring additional clinical benefits to embolization. This study aims to answer this question by investigating HCC patient outcomes after TACE treatment using Embozene or Embosphere as the embolic agent. Patient outcomes, including liver function, tumor size, tumor response, and complications after TACE treatment are compared between these two treatments.

\section{Materials and Methods}

\section{Patient cohort}

This retrospective study includes HCC patients who received TACE with Embozene or Embosphere as the embolic agent at a single medical center between May 2014 and September 2016. The cohort comprised a total of 108 patients, 38 who received Embosphere 100-300 $\mu \mathrm{m}$ (Merit Medical, South Jordan, UT) (group A) and 70 who received Embozene 75 $\mu \mathrm{m}, 100 \mu \mathrm{m}$, or $250 \mu \mathrm{m}$ (Celonova Biosciences, Inc., Atlanta, GA) (group B). All included patients had at least one treatment-naïve lesion $\geq 10 \mathrm{~mm}$, Child-Pugh score $\leq 8$, serum bilirubin $\leq 2$, not amenable for operation or radiofrequency ablation, and no or mild ascites. To avoid the systemic effect of chemical, targeted, or immunotherapy drugs on the untreated target lesion, patients who received these drugs were excluded.

Data were gathered from patient medical records, including demographic data, history of hepatitis virus infection, Child-Pugh score, and duration of hospitalization. All patients had baseline and 1-3-month post-treatment images. We used the modified Response Evaluation Criteria in Solid Tumors (mRECIST) to evaluate target lesions.

\section{TACE implementation}

\section{1) Procedure}

The therapeutic plan for each patient was devised by their physician, including the timing of TACE implementation and choice of microspheres. Femoral puncture was performed and a 5-Fr angiosheath (Medikit, Tokyo, Japan) was placed in the artery. A 4- or 5-Fr angiocatheter was selected by the radiologist for further procedure. Digital subtraction angiography (DSA) at the celiac trunk was performed in every patient. TACE was performed using a microcatheter and super selection technique to approach the target lesion. To control vasospasm, intra-arterial nitroglycerin was infused at a dose of $\leq 250 \mu$ g. An emulsion of lipiodol, a vehicle that carries the drug into the tumor, and doxorubicin (Embosphere: $1 \mathrm{~mL}$ lipiodol to $5 \mathrm{mg}$ doxorubicin; Embozene, $1 \mathrm{~mL}$ lipiodol to 2.5 $\mathrm{mg}$ doxorubicin) was then infused with the embolic agent according to the manufacturer's instructions. The endpoint of embolization was the point at which the test contrast column in the proximal hepatic artery was clear within five patient heartbeats. The post-TACE DSA was evaluated using halfdose contrast. If the tumor stain was still visible, the embolization was repeated until the endpoint was reached.

\section{2) Dose determination}

We presume that a more size-uniform microsphere suspension is less porous and more compact, thereby size-uniform microspheres would lead to a more distal occlusion to target area than less uniform microspheres. Thus, the administrating amount of lipiodol were determined by the type of embolic agent used (Embosphere: $1 \mathrm{~mL}$ lipiodol for $1 \mathrm{~cm}$ target area; Embozene, $0.5 \mathrm{~mL}$ lipiodol for $1 \mathrm{~cm}$ target area). The administrating dose of chemotherapeutic agents were depended on the amount of lipiodol, as $5 \mathrm{mg}$ chemotherapeutic agent emulsion/mL lipiodol. For example, a 2-cm diameter target 
Table 1. Baseline patient characteristics according to the treatment group

\begin{tabular}{|c|c|c|c|c|}
\hline & $\begin{array}{c}\text { Total } \\
(n=108)\end{array}$ & $\begin{array}{l}\text { Embosphere } \\
\qquad(n=38)\end{array}$ & $\begin{array}{c}\text { Embozene } \\
(n=70)\end{array}$ & p-value ${ }^{a)}$ \\
\hline Age (yr) & $67.07 \pm 9.86$ & $68.39 \pm 9.28$ & $66.36 \pm 10.16$ & 0.307 \\
\hline \multicolumn{5}{|l|}{ Sex } \\
\hline Female & $40(37.0)$ & $12(31.6)$ & $28(40.0)$ & 0.413 \\
\hline Male & $68(63.0)$ & $26(68.4)$ & $42(60.0)$ & \\
\hline \multicolumn{5}{|l|}{$\mathrm{HBV} / \mathrm{HCV}$} \\
\hline None & $10(9.3)$ & $4(10.5)$ & $6(8.6)$ & 0.595 \\
\hline HBV & $50(46.3)$ & $17(44.7)$ & $33(47.1)$ & \\
\hline $\mathrm{HCV}$ & $45(41.7)$ & $17(44.7)$ & $28(40.0)$ & \\
\hline $\mathrm{HBV}+\mathrm{HCV}$ & $3(2.8)$ & 0 & $3(4.3)$ & \\
\hline \multicolumn{5}{|c|}{ Child-Pugh score } \\
\hline A & $99(91.7)$ & $36(94.7)$ & $63(90.0)$ & 0.489 \\
\hline B & $9(8.3)$ & $2(5.3)$ & $7(10.0)$ & \\
\hline \multicolumn{5}{|l|}{ BCLC staging } \\
\hline A & $5(4.6)$ & $1(2.6)$ & $4(5.7)$ & 0.655 \\
\hline B & $103(95.4)$ & $37(97.4)$ & $66(94.3)$ & \\
\hline
\end{tabular}

Values are presented as the mean \pm standard deviation and number $(\%)$. HBV, hepatitis B virus; $\mathrm{HCV}$, hepatitis $\mathrm{C}$ virus; $\mathrm{BCLC}$, Barcelona Clinic Liver Cancer. ${ }^{a}$ Embosphere vs. Embozene as determined by two-sample $t$ test for age and Pearson chisquare/ or Fisher exact test for other categorical variables. No significant differences were observed in baseline characteristics between the two treatment groups.

Table 2. Laboratory results according to treatment

\begin{tabular}{|c|c|c|c|c|}
\hline & $\begin{array}{c}\text { Total } \\
(n=108)\end{array}$ & $\begin{array}{c}\text { Embosphere } \\
(n=38)\end{array}$ & $\begin{array}{l}\text { Embozene } \\
\qquad(n=70)\end{array}$ & p-value ${ }^{a}$ \\
\hline \multicolumn{5}{|l|}{ GOT (U/L) } \\
\hline Ratio $^{\text {b) }}$ & $177.99 \pm 234.31$ & $147.49 \pm 174.51$ & $194.54 \pm 260.8$ & $0.032^{\mathrm{c})}$ \\
\hline Pre-treatment & $70.51 \pm 43.58$ & $67.32 \pm 33.21$ & $72.24 \pm 48.43$ & 0.748 \\
\hline Post-treatment & $116.31 \pm 168.21$ & $90.10 \pm 102.24$ & $130.54 \pm 194.14$ & 0.257 \\
\hline \multicolumn{5}{|l|}{ GPT (U/L) } \\
\hline Ratio $^{\text {b) }}$ & $173.03 \pm 164.49$ & $155.74 \pm 118.25$ & $182.42 \pm 184.95$ & 0.286 \\
\hline Pre-treatment & $61.22 \pm 53.76$ & $54.26 \pm 32.53$ & $65.00 \pm 62.23$ & 0.632 \\
\hline Post-treatment & $87.67 \pm 77.87$ & $74.50 \pm 60.42$ & $94.81 \pm 85.44$ & 0.465 \\
\hline \multicolumn{5}{|l|}{ Bilirubin (mg/dL) } \\
\hline Ratio $^{\text {b) }}$ & $147.1 \pm 116.61$ & $125.93 \pm 61.78$ & $158.6 \pm 136.6$ & 0.117 \\
\hline Pre-treatment & $0.72 \pm 0.42$ & $0.62 \pm 0.25$ & $0.78 \pm 0.48$ & 0.122 \\
\hline Post-treatment & $0.97 \pm 0.76$ & $0.73 \pm 0.37$ & $1.11 \pm 0.88$ & $0.016^{c)}$ \\
\hline \multicolumn{5}{|l|}{ Creatinine (mg/dL) } \\
\hline Ratio $^{\text {b) }}$ & $99.24 \pm 24.88$ & $96.79 \pm 12.75$ & $100.56 \pm 29.46$ & 0.560 \\
\hline Pre-treatment & $1.13 \pm 1.27$ & $1.05 \pm 0.70$ & $1.18 \pm 1.50$ & 0.256 \\
\hline Post-treatment & $1.16 \pm 1.54$ & $1.03 \pm 0.78$ & $1.23 \pm 1.82$ & 0.294 \\
\hline
\end{tabular}

Values are presented as the mean \pm standard deviation. GOT, glutamate oxaloacetate transaminase; GPT, glutamate pyruvate transaminase. ${ }^{a}$ Embosphere vs. Embozene as determined by Mann-Whitney U test, ${ }^{b}$ Ratio=Post-treatment / Pre-treatment, c)Significant difference. 
Table 3. Patient outcomes according to treatment

\begin{tabular}{|c|c|c|c|c|}
\hline & $\begin{array}{c}\text { Total } \\
(n=108)\end{array}$ & $\begin{array}{l}\text { Embosphere } \\
\qquad(n=38)\end{array}$ & $\begin{array}{c}\text { Embozene } \\
(n=70)\end{array}$ & p-value ${ }^{a)}$ \\
\hline Duration of hospitalization & $\begin{array}{c}3.86 \pm 2.48 \\
\quad(2-24)\end{array}$ & $\begin{array}{c}3.95 \pm 2.03 \\
\quad(2-11)\end{array}$ & $\begin{array}{r}3.81 \pm 2.7 \\
(2-24)\end{array}$ & 0.786 \\
\hline \multicolumn{5}{|l|}{ Chemical agent } \\
\hline TAE & $7(6.5)$ & 0 & $7(10.0)$ & 0.050 \\
\hline TACE & $101(93.5)$ & $38(100)$ & $63(90.0)$ & \\
\hline Lipiodol (mL) & $\begin{array}{c}3.45 \pm 2.86 \\
\quad(1-12)\end{array}$ & $\begin{array}{c}5.97 \pm 2.93 \\
\quad(1-12)\end{array}$ & $\begin{array}{c}2.08 \pm 1.64 \\
\quad(1-10)\end{array}$ & $<0.001^{\mathrm{b})}$ \\
\hline Doxorubicin (mg) & $\begin{array}{c}17.23 \pm 4.31 \\
(5-60)\end{array}$ & $\begin{array}{c}29.84 \pm 14.64 \\
(5-60)\end{array}$ & $\begin{array}{c}10.38 \pm 8.22 \\
(5-50)\end{array}$ & $<0.001^{\mathrm{b})}$ \\
\hline No. of target lesions & $2.29 \pm 1.29$ & $2.87 \pm 1.19$ & $1.97 \pm 1.24$ & $<0.001$ \\
\hline 1 & $40(37)$ & $6(15.8)$ & $34(48.6)$ & \\
\hline 2 & $26(24.1)$ & $8(21.1)$ & $18(25.7)$ & \\
\hline 3 & $22(20.4)$ & $12(31.6)$ & $10(14.3)$ & \\
\hline 4 & $11(10.2)$ & $9(23.7)$ & $2(2.9)$ & \\
\hline 5 & $9(8.3)$ & $3(7.9)$ & $6(8.6)$ & \\
\hline \multicolumn{5}{|c|}{ Total size of target lesions (mm) } \\
\hline Pre-treatment & $47.99 \pm 34.59$ & $56.37 \pm 25.91$ & $43.44 \pm 37.89$ & $0.001^{b)}$ \\
\hline Post-treatment & $23.6 \pm 23.73$ & $31.03 \pm 18.14$ & $19.57 \pm 25.5$ & $<0.001^{\mathrm{b})}$ \\
\hline Change $(\%)$ & $46.75 \pm 35.03$ & $55.86 \pm 25.55$ & $41.81 \pm 38.51$ & $0.027^{\mathrm{b})}$ \\
\hline \multicolumn{5}{|c|}{ Progression of non-target lesions } \\
\hline No & $99(91.7)$ & $36(94.7)$ & $63(90.0)$ & 0.489 \\
\hline Yes & $9(8.3)$ & $2(5.3)$ & $7(10.0)$ & \\
\hline \multicolumn{5}{|l|}{ Response } \\
\hline CR & $22(20.4)$ & $1(2.6)$ & $21(30.0)$ & $0.002^{\mathrm{b})}$ \\
\hline PR & $52(48.2)$ & $24(63.2)$ & $28(40.0)$ & \\
\hline SD & $25(23.2)$ & $11(29)$ & $14(20.0)$ & \\
\hline PD & $9(8.3)$ & $2(5.3)$ & $7(10.0)$ & \\
\hline Major complications & $4(3.7)$ & $2(5.3)$ & $2(2.9)$ & 0.612 \\
\hline Minor complications & $20(18.5)$ & $11(28.9)$ & $9(12.9)$ & $0.040^{\mathrm{b})}$ \\
\hline
\end{tabular}

Values are presented as the mean \pm standard deviation (range) or number (\%). TAE, transarterial embolization; TACE, transarterial chemoembolization; CR, complete remission; PR, partial response; SD, stable disease; PD, progressing disease. a)Embosphere vs. Embozene as determined by Mann-Whitney U test for continuous variables and Pearson chi-square or Fisher exact test for categorical variables, ${ }^{\text {b) }}$ Significant difference.

area were treated by $2 \mathrm{~mL}$ of lipiodol containing $10 \mathrm{mg}$ doxorubicin. The patient with a 3-cm diameter target area who were treated with Embozene receiving $1.5 \mathrm{~mL}$ lipiodol containing $7.5 \mathrm{mg}$ doxorubicin.

Triple-phase contrast-enhanced computed tomography or contrast-enhanced magnetic resonance was performed at baseline and during post-therapeutic evaluation at 1-3 months. The images were interpreted by two radiologists and evaluated using mRECIST criteria, as follows: complete remission (CR), absence of intratumoral arterial enhancement in all target lesions; partial response (PR), decreased sum of the diameter of viable target lesions; stable disease (SD), no PR and no progressing disease (PD); PD, increase of $>20 \%$ in the sum of the smallest diameter target lesions since the start of the treatment. Major complications included fever, abdominal pain, and other adverse events necessitating the use of medication or further management. Minor complications included nausea, vomiting, fever, abdominal pain, and other adverse events that did not require medication or further management.

\section{Statistical analysis}

Data are presented as the mean \pm standard deviation (SD) for age, laboratory examinations, and the number of target lesions; mean $\pm S D$ (range) for length of hospital stay and dosage of lipiodol and doxorubicin; and number (\%) for 
Table 4. Change in total size of target lesions and tumor response $(\mathrm{CR}+\mathrm{PR})$ according to treatment and patient characteristics

\begin{tabular}{|c|c|c|c|c|}
\hline & \multicolumn{2}{|c|}{ Change of total size of target lesions $(\%)^{a)}$} & \multicolumn{2}{|c|}{ Tumor response $(\mathrm{CR}+\mathrm{PR})^{\mathrm{b})}$} \\
\hline & $\boldsymbol{\beta}(95 \% \mathrm{CI})$ & p-value & OR $(95 \% \mathrm{CI})$ & p-value \\
\hline \multicolumn{5}{|l|}{ Embolic agent } \\
\hline Embosphere & Reference & & 1 & \\
\hline Embozene & $-14.05(-27.84$ to -0.25$)$ & $0.046^{\mathrm{c})}$ & $1.21(0.52$ to 2.82$)$ & 0.653 \\
\hline Age (yr) & $0.08(-0.61$ to 0.76$)$ & 0.827 & $0.99(0.95$ to 1.03$)$ & 0.666 \\
\hline \multicolumn{5}{|l|}{ Sex } \\
\hline Female & Reference & & 1 & \\
\hline Male & $5.75(-8.11$ to 19.61$)$ & 0.413 & $0.49(0.20$ to 1.22$)$ & 0.127 \\
\hline \multicolumn{5}{|l|}{ Chemical agent } \\
\hline TAE & Reference & & 1 & \\
\hline TACE & $6.40(-20.84$ to 33.65$)$ & 0.642 & $0.34(0.04$ to 2.97$)$ & 0.332 \\
\hline TACE with Lipiodol+Doxorubicin & $2.48(0.003$ to 4.95$)$ & $0.050^{\text {c) }}$ & $0.45(0.05$ to 4.19$)$ & 0.483 \\
\hline \multicolumn{5}{|l|}{$\mathrm{HBV} / \mathrm{HCV}$} \\
\hline None & Reference & & 1 & \\
\hline HBV & $3.81(-20.44$ to 28.07$)$ & 0.723 & 0.48 (0.09 to 2.54$)$ & 0.392 \\
\hline $\mathrm{HCV}$ & $-4.39(-28.87$ to 20.09$)$ & 0.756 & $0.55(0.10$ to 2.95$)$ & 0.488 \\
\hline $\mathrm{HBV}+\mathrm{HCV}$ & $-5.24(-51.33$ to 40.85$)$ & 0.822 & $0.50(0.03$ to 8.71$)$ & 0.634 \\
\hline \multicolumn{5}{|l|}{ Child-Pugh score } \\
\hline A & Reference & & 1 & \\
\hline B & $-8.2(-32.44$ to 16.04$)$ & 0.504 & $0.91(0.21$ to 3.88$)$ & 0.901 \\
\hline No. of target lesions & $2.89(-2.3$ to 8.09$)$ & 0.272 & $0.87(0.64$ to 1.19$)$ & 0.399 \\
\hline Pre-treatment total size of target lesions (mm) & $0.098(-0.096$ to 0.292$)$ & 0.318 & $1.00(0.99$ to 1.01$)$ & 0.827 \\
\hline Change in total size of target tumors $(\%)$ & $\mathrm{n} / \mathrm{a}$ & - & $0.91(0.88$ to 0.95$)$ & $<0.001^{\text {c) }}$ \\
\hline
\end{tabular}

CR, completed response; PR, partial response; CI, confidence interval; OR, odds ratio; TAE, transarterial embolization; TACE,

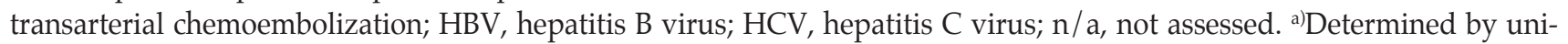
variate linear regression analysis, ${ }^{b}$ Determined by logistic regression analysis, ${ }^{c}$ Significant difference.

other categorical variables by treatment. Differences between the two treatments were evaluated using a two-sample $t$ test for continuous data, the Mann-Whitney $U$ test for continuous variables without normal distribution, and the Pearson chisquare or Fisher exact test for categorical variables. Univariate simple linear regression and binary logistic regression analyses were applied to evaluate the association of $\%$ change in total size of target lesions and tumor response $(\mathrm{CR}+\mathrm{PR})$ according to treatment and clinical characteristics, separately. Results are presented as $\beta$ with corresponding $95 \%$ confidence interval $(95 \% \mathrm{CI})$ and p-values via univariate linear regression analysis and as odds ratio (OR) with corresponding 95\% CI and p-value via binary logistic regression analysis. All statistical assessments were two-tailed and considered significant for $p<0.05$. All statistical analyses were performed using IBM SPSS statistical software ver. 22 for Windows (IBM Corp., Armonk, NY).

\section{Ethical statement}

This study was approved by the Institutional Review Board of National Cheng Kung University Hospital with a waiver of informed consent (IRB No. B-ER-106-020) and performed in accordance with the principles of the Declaration of Helsinki.

\section{Results}

The patient cohort comprised a total of 108 patients, 38 treated with Embosphere and 70 treated with Embozene. The mean age of all patients was 67.07 years (SD, 9.86). The majority of patients were male $(63 \%)$, and $90.7 \%$ were infected with hepatitis B virus, hepatitis C virus, or both. Tumor and liver function assessment classified $91.7 \%$ of the patients as stage A according to Child-Pugh score and $95.4 \%$ as stage B 
according to Barcelona Clinic Liver Cancer classification (Table 1).

Laboratory results show that the glutamate oxaloacetate transaminase (GOT) ratio was higher in the Embozene group than in the Embosphere group (mean, 194.5 vs. 147.5; $\mathrm{p}=0.032$ ). The postoperative bilirubin level was higher in the Embozene group than in the Embosphere group (mean, $1.11 \mathrm{mg}$ / $\mathrm{dL}$ vs. $0.73 \mathrm{mg} / \mathrm{dL} ; \mathrm{p}=0.016$ ) (Table 2). On average, the duration of hospital stay for all patients after treatment was 3.86 days (range, 2 to 24 days). A TACE chemical agent was administered to all patients in the Embosphere group and 90\% of those in the Embozene group (Table 3).

Before treatment, the average number of target lesions was higher in the Embosphere group than in the Embozene group $(2.87 \pm 1.19$ vs. $1.97 \pm 1.24, \mathrm{p}<0.001)$, and the total target tumor size was larger in the Embosphere group $(56.37 \pm 25.91$ $\mathrm{mm}$ vs. $43.44 \pm 37.89 \mathrm{~mm}, \mathrm{p}=0.001$ ). After treatment, the total target tumor decreased in both groups, with the percentage change greater in the Embosphere group than in the Embozene group ( $55.86 \pm 25.55 \%$ vs. $41.81 \pm 38.51 \%$, $\mathrm{p}=0.027)$. Two months after treatment, the tumor response differed significantly between the groups ( $p=0.002$ ), while the $C R+P R$ response rate did not differ significantly between the groups (65.8\% vs. $70 \%, \mathrm{p}=0.653$ ) (data not shown). Four patients $(3.7 \%)$ had major complications, and 20 patients $(18.5 \%)$ had minor complications after treatment. The occurrence rate of minor complications was higher in the Embosphere group than in the Embozene group (28.9\% vs. $12.9 \%$, $\mathrm{p}=0.040$ ) (Table 3 ).

Univariate analysis results show that patients treated with Embozene experienced a greater percentage change in total tumor size $(\beta=-4.05, p=0.046)$ than did those treated with Embosphere. A marginal association was found between the percentage change in total tumor size and lipiodol+doxorubicin combination treatment $(\mathrm{p}=0.050$ ) (Table 4). Multivariate analysis showed no significant associations with lipiodol+ doxorubicin treatment (data not shown). Regarding tumor response, the only significant association of $\mathrm{CR}+\mathrm{PR}$ was with the percentage change in total size of target lesions, by univariate logistic regression analysis $(\mathrm{OR}, 0.91 ; \mathrm{p}<0.001)$ (Table 4).

\section{Discussion}

This study compares the efficacy of the embolic agents Embozene and Embosphere for use with TACE in HCC patients. We observed that while postoperative GOT and bilirubin were higher among patients treated with Embozene, the decrease in the number and size of liver tumors relative to baseline was greater in these patients than in those treated with Embosphere. Furthermore, these greater effects on tumors were achieved using lower doses of the anticancer drug doxorubicin than in treatment with Embozene. The occurrence rate of minor complications was higher among patients treated with Embosphere than with Embozene. These results suggest that Embozene is more efficacious than Embosphere for HCC treatment using TACE/lipiodol-doxorubicin.

The greater tumor effects of Embozene are consistent with the known characteristics of this product. A previous study in sheep reports that the particle size is more precisely calibrated in Embozene than in Embosphere, allowing it to penetrate further into distal vessels [9]. Tight calibration of particles within a small range of diameters is important to eliminate larger particles that can occlude microvessels more proximally, which prevents deeper penetration of smaller particles during administration [10]. In addition, the deformation of Embozene is greater than that of Embosphere and appears to determine the size (i.e., distality) of the vessel occluded as opposed to the in vitro granulometric particle size [9].

We observed that the better outcome by Embozene was achieved using lower doses of doxorubicin than that administered with Embosphere. This result might arise from the deeper distal occlusion of vessels by Embozene, maximizing the vascular shutdown of the tumor and achievement of an anoxic environment. The vascular occlusion induced by TACE increases the cytotoxic effect over that of doxorubicin alone [11]; thus, maximization of vascular occlusion may result in greater cytotoxicity at lower drug doses. This factor may be of importance to patients experiencing adverse reactions to doxorubicin, which is reported to cause occasional acute dyspnea associated with transient neutropenia during systemic infusion [12]. The use of Embozene may allow for suitable tumor effects with less drug administered.

This study has several limitations. First, the retrospective design of the study makes it subject to bias and confounding and precludes the determination of cause and effect. Second, this study only investigates medium- to large-sized particles. We also lack the small particle granulometry to strengthen our theory. Third, TACE with Embosphere is in the early stages of development, so the proficiency of this technique should be considered in viewing these results. The small sample size also is a limitation of this study.

The use of Embozene as the embolic agent in TACE provides a better target tumor response at lower doses of chemomedication, possibly because of its more precisely-sized microspheres. Future studies addressing granulometry in relation to tumor response may provide more solid evidence to support this conclusion.

\section{Conflicts of Interest}

Conflict of interest relevant to this article was not reported. 


\section{References}

1. Balogh J, Victor D 3rd, Asham EH, Burroughs SG, Boktour M, Saharia A, et al. Hepatocellular carcinoma: a review. J Hepatocell Carcinoma. 2016;3:41-53.

2. Wang YX, De Baere T, Idee JM, Ballet S. Transcatheter embolization therapy in liver cancer: an update of clinical evidences. Chin J Cancer Res. 2015;27:96-121.

3. Lencioni R, Petruzzi P, Crocetti L. Chemoembolization of hepatocellular carcinoma. Semin Intervent Radiol. 2013;30:311.

4. Bruix J, Sherman M; Practice Guidelines Committee, American Association for the Study of Liver Diseases. Management of hepatocellular carcinoma. Hepatology. 2005;42:1208-36.

5. Llovet JM, Di Bisceglie AM, Bruix J, Kramer BS, Lencioni R, Zhu AX, et al. Design and endpoints of clinical trials in hepatocellular carcinoma. J Natl Cancer Inst. 2008;100:698-711.

6. Marelli L, Stigliano R, Triantos C, Senzolo M, Cholongitas E, Davies N, et al. Transarterial therapy for hepatocellular carcinoma: which technique is more effective? A systematic review of cohort and randomized studies. Cardiovasc Intervent Radiol. 2007;30:6-25.

7. Lewis AL, Adams C, Busby W, Jones SA, Wolfenden LC, Lep- pard SW, et al. Comparative in vitro evaluation of microspherical embolisation agents. J Mater Sci Mater Med. 2006;17:1193204.

8. Vaidya S, Tozer KR, Chen J. An overview of embolic agents. Semin Intervent Radiol. 2008;25:204-15.

9. Verret V, Ghegediban SH, Wassef M, Pelage JP, Golzarian J, Laurent A. The arterial distribution of Embozene and Embosphere microspheres in sheep kidney and uterus embolization models. J Vasc Interv Radiol. 2011;22:220-8.

10. Bonomo G, Pedicini V, Monfardini L, Della Vigna P, Poretti D, Orgera G, et al. Bland embolization in patients with unresectable hepatocellular carcinoma using precise, tightly sizecalibrated, anti-inflammatory microparticles: first clinical experience and one-year follow-up. Cardiovasc Intervent Radiol. 2010;33:552-9.

11. Facciorusso A, Licinio R, Muscatiello N, Di Leo A, Barone M. Transarterial chemoembolization: Evidences from the literature and applications in hepatocellular carcinoma patients. World J Hepatol. 2015;7:2009-19.

12. Vahid B, Marik PE. Pulmonary complications of novel antineoplastic agents for solid tumors. Chest. 2008;133:528-38. 